\author{
SERIES "CONTRIBUTIONS FROM THE EUROPEAN RESPIRATORY MONOGRAPH" \\ Edited by M. Decramer and A. Rossi \\ Number 10 in this Series
}

\title{
Prone position in acute respiratory distress syndrome
}

\author{
P. Pelosi*, L. Brazzi ${ }^{\#}$ L. Gattinoni ${ }^{\#}$
}

\begin{abstract}
Prone position in acute respiratory distress syndrome. P. Pelosi, L. Brazzi, L. Gattinoni. C) ERS Journals Ltd 2002.

ABSTRACT: In the last few years prone positioning has been used increasingly in the treatment of patients with acute respiratory distress syndrome (ARDS) and this manoeuvre is now considered a simple and safe method to improve oxygenation. However, the physiological mechanisms causing respiratory function improvement as well as the real clinical benefit are not yet fully understood. The aim of this review is to discuss the physiological and clinical effects of prone positioning in patients with ARDS.

The main physiological aims of prone positioning are: 1) to improve oxygenation; 2) to improve respiratory mechanics; 3 ) to homogenise the pleural pressure gradient, the alveolar inflation and the ventilation distribution; 4) to increase lung volume and reduce the amount of atelectatic regions; 5) to facilitate the drainage of secretions; and 6) to reduce ventilator-associated lung injury.

According to the available data, the authors conclude that: 1) oxygenation improves in $\sim 70-80 \%$ of patients with early acute respiratory distress syndrome; 2 ) the beneficial effects of oxygenation reduce after 1 week of mechanical ventilation; 3) the aetiology of acute respiratory distress syndrome may markedly affect the response to prone positioning; 4) extreme care is necessary when the manoeuvre is performed; 5 ) pressure sores are frequent and related to the number of pronations; 6 ) the supports used to prone and during positioning are different and nonstandardised among centres; and 7) intensive care unit and hospital stay and mortality still remain high despite prone positioning. Eur Respir J 2002; 20: 1017-1028.
\end{abstract}

*Dept of Scienze Cliniche e Biologiche, Universita' degli Studi dell'Insubria, Varese, and \#Istituto di Anestesia e Rianimazione, Universita' degli Studi di Milano, Ospedale Maggiore Policlinico, IRCCS, Milan, Italy.

Correspondence: L. Gattinoni

Istituto di Anestesia e Rianimazione Via F. Sforza 35

20122

Milan

Italy

Keywords: Acute respiratory distress syndrome

intensive care

oxygenation

prospective studies

Received: March 182002

Accepted: March 212002
Acute respiratory distress syndrome (ARDS) is characterised by radiographical diffuse bilateral infiltrates, decreased respiratory compliance, small lung volumes and severe hypoxaemia. Correction of lifethreatening hypoxia and improvement of respiratory mechanics and lung volumes are the main treatment goals. To achieve these ends, it is important to select the most appropriate means of ventilatory support, thereby minimising the damaging effects of mechanical ventilation. Currently, ventilatory support using small tidal volumes and low plateau pressures and respiratory rate, to control arterial carbon dioxide tension $\left(P \mathrm{a}, \mathrm{CO}_{2}\right)$ and $\mathrm{pH}$, are considered optimal [1]. Moreover, the application of relatively high levels of positive end-expiratory pressure (PEEP) seems to be beneficial in reducing ventilator-associated lung injury (VALI) and improving survival [2]. In 1974, BRYAN [3] suggested that anaesthetised and paralysed patients in the prone position should exhibit better expansion of the dorsal lung regions with a consequent improvement in oxygenation. In 1976, PIEHL and BROWN [4] showed, in a retrospective study, that the prone position improved oxygenation in five patients with ARDS without deleterious effects. One year later, Douglas et al. [5] demonstrated, in a prospective study with a limited group of ARDS patients, that prone positioning could effectively improve oxygenation in ARDS. Starting from these reports, interest in prone positioning in ARDS has increased progressively and it is now considered to be a simple and safe method of improving oxygenation in severely hypoxaemic patients [6]. However, the physiological mecha nisms that cause improvement in oxygenation are not fully understood. Randomised clinical studies showing a reduction in morbidity and mortality are lacking, and turning from the supine to prone position is

Previous articles in this series: No. 1: Baldacci S, Omenaas E, Oryszczyn MP. Allergy markers in respiratory epidemiology. Eur Respir 2001; 17: 773-790. No. 2: Antó JM, Vermeire P, Vestbo J, Sunyer J. Epidemiology of chronic obstructive pulmonary disease. Eur Respir J 2001; 17: 982-994. No. 3: Cuvelier A, Muir J-F. Noninvasive ventilation and obstructive lung diseases. Eur Respir J 2001; 17: 1271-1281. No. 4: Wysocki M, Antonelli M. Noninvasive mechanical ventilation in acute hypoxaemic respiratory failure. Eur Respir J 2001; 18: 209-220. No. 5: Østerlind K. Chemotherapy in small cell lung cancer. Eur Respir J 2001; 18: 1026-1043. No. 6: Jaakkola MS. Environmental tobacco smoke and health in the elderly. Eur Respir J 2002; 19: 172-181. No. 7: Hollings N, Shaw P. Diagnostic imaging of lung cancer. Eur Respir J 2002; 19: 722-742. No. 8: Künzli N. The public health relevance of air pollution abatement. Eur Respir J 2002; 20: 198-209. No. 9: D'Amato G, Liccardi G, D'Amato M, Cazzola M. Outdoor air pollution, climatic changes and allergic bronchial asthma. Eur Respir J 2002; 20: 763-776. 
still considered a complex clinical procedure associated with potential life-threatening complications. This review aims to discuss the physiological basis of the beneficial effects of prone positioning and its most relevant effects in patients with ARDS.

\section{Physiological effects of prone positioning}

The physiological effects of prone positioning in patients with severe lung injury manifest as improvements in oxygenation and respiratory mechanics. There may also be a reduction in mechanical factors associated with VALI, such as an inhomogeneous distribution of pleural pressure $(P \mathrm{pl})$, alveolar inflation and ventilation, an increase in lung volume and reduction in atelectatic lung regions and, finally, an improvement in clearance of secretions. Since the majority of animal and human studies investigating the physiological effects of prone positioning refer to acute ARDS, the considerations discussed later apply only to the early stages of the syndrome.

\section{Effects on oxygenation}

Before describing the mechanisms by which the prone position improves oxygenation, the physiology of the distribution of alveolar inflation, alveolar ventilation and perfusion is discussed.

Distribution of alveolar inflation in the supine position. Figure 1 shows the distribution of alveolar inflation (expressed as the gas/tissue ratio) in the supine and prone positions in normal subjects and patients with ARDS. In normal subjects, the distribution of alveolar inflation follows a gravitational gradient, the nondependent alveoli, located near to the sternum, being more distended than the dependent ones posteriorly. Alveolar dimensions depend on the transpulmonary pressure, the difference in pressure

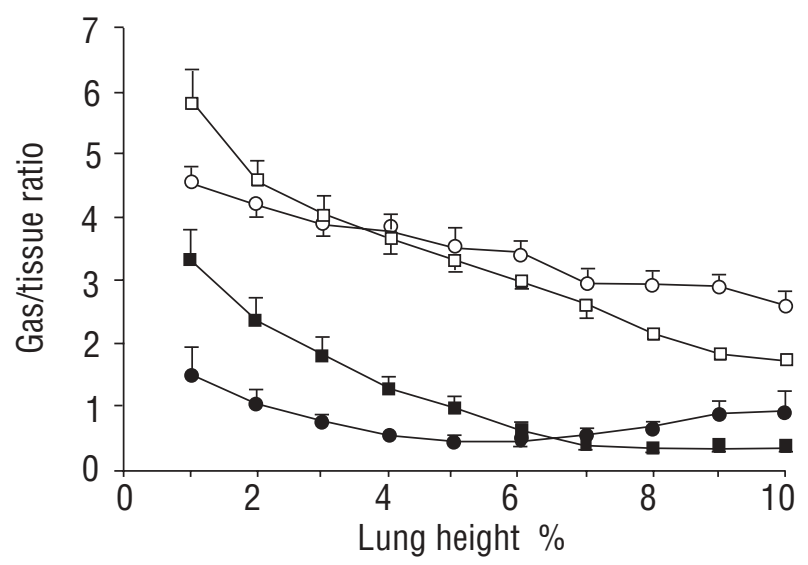

Fig. 1.- The gas/tissue ratio, an index of alveolar inflation in normal lung $(\mathrm{n}=14)$, supine $(\square)$ and prone $(\bigcirc)$ position, and lung of acute respiratory distress syndrome patients $(n=20)$, supine $(\boldsymbol{\square})$ and prone $(\bullet)$ position. A height of $0 \%$ refers to the ventral surface in supine position and to the dorsal surface in prone position. Reproduced with permission from GATTINONI et al. [7]. between the alveolar pressure $(P A)$ and $P$ pl. Since $P$ A is more negative in nondependent lung regions, transpulmonary pressure is greater in the nondependent, compared to the dependent areas. The nature of the transpulmonary pressure gradient is unclear, but has been attributed to several factors such as the effects of lung weight, cardiac mass, cephalic displacement of the diaphragm, the regional shape and mechanical properties of the chest wall and lungs.

Lung weight. In ARDS, the lung is characterised by radiographic densities, primarily located in dependent regions (fig. 2). Regional analysis of the lung using computed tomography (CT) has revealed that alveolar inflation is reduced markedly both in the ventral (near the sternum in supine position) and dorsal regions, following a gravitational gradient [8] (fig. 1). Thus, the nondependent alveoli are relatively more expanded than the dependent ones. By contrast, the distribution of oedema is uniform throughout the lung parenchyma suggesting that the disease process is distributed uniformly. As the total mass of the lung is increased, it progressively collapses under its own weight, squeezing out gas from the dependent lung regions causing compression atelectasis.

Cardiac mass. In normal subjects, the weight of the heart on dependent regions has a significant influence on the aeration of the subjacent lung. Experimental evidence suggests that the heart contributes to the genesis of the vertical gradient of transpulmonary pressure under physiological conditions [9]. In patients with ARDS the cardiac mass is increased compared with normal subjects, resulting in increased $P \mathrm{pl}$, in the dependent part of the lung, and alveolar collapse [10].

Cephalic displacement of the abdomen. Sedation and paralysis suppress diaphragmatic muscular tone. The weight of the abdominal contents, no longer opposed by the diaphragm, induces a cephalic displacement of posterior regions of the diaphragm [11]. This upward shift increases $P \mathrm{pl}$ in the most caudal and dependent diaphragmatic regions, contributing to the formation of basal atelectasis.

Regional lung and chest wall mechanical properties and shape. Regional lung and chest wall mechanical properties may influence regional transpulmonary pressure [12]. In particular, the distensibility of the lung (which, in turn, depends on lung volume and contents), and the distensibility of the thorax (which depends on chest wall mass, compliance and perhaps the anatomy of the diaphragm) may play a role. Moreover, the shape of the lung and chest wall may influence regional transpulmonary pressure. It is clear that $P \mathrm{pl}$ depends on the relationships between the external box (the chest wall and, in particular, the thoracic cage) and the content (the lung). A thoracic shape (chest wall and lung) more similar to a triangle in the supine position (apex on top) allows the formation of more extensive atelectasis than a rectangular thoracic shape [7].

To conclude, in ARDS patients in the supine 

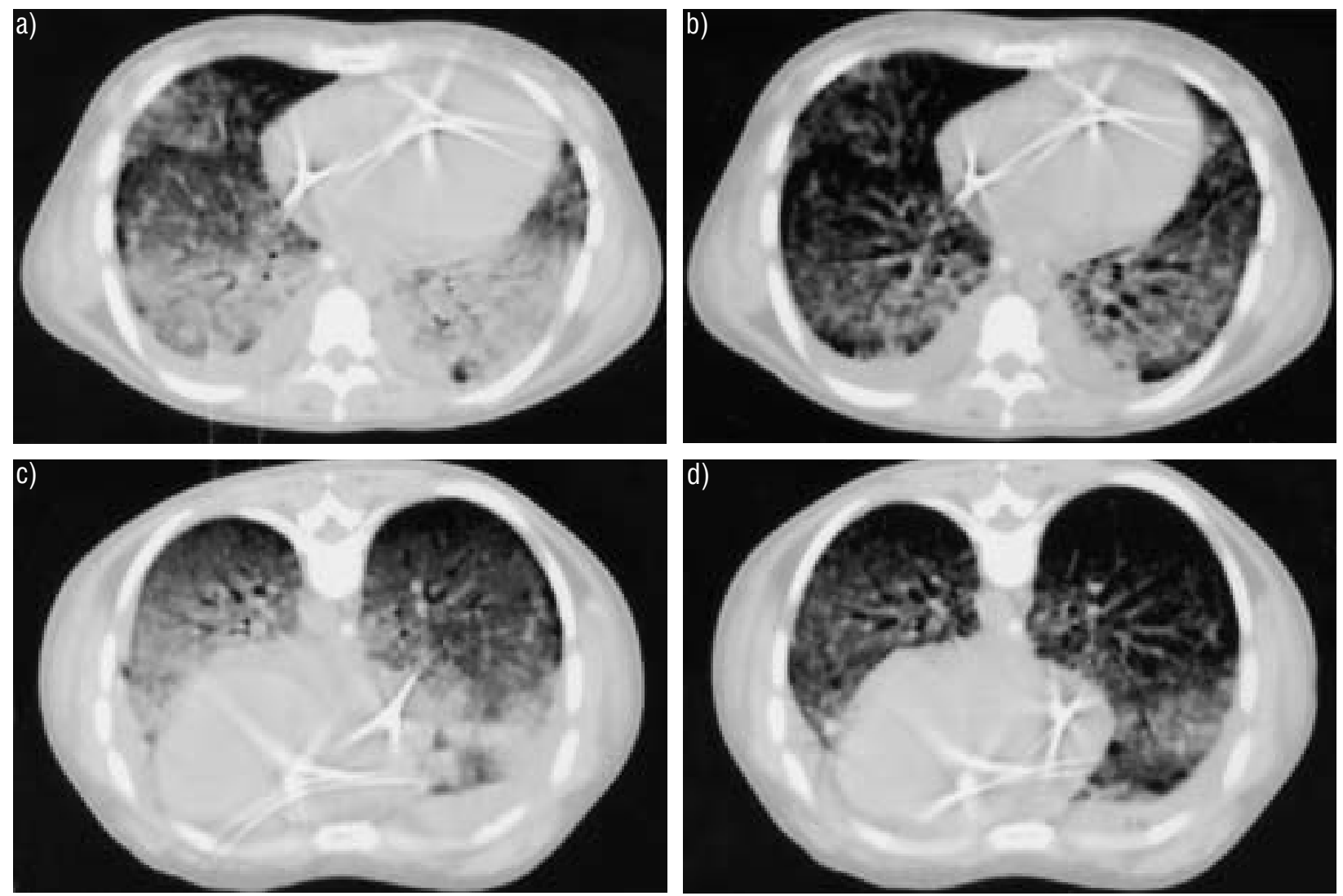

Fig. 2. - Effects on lung densities of supine positioning at a) end-expiration and at b) end-inspiration, and prone positioning at c) endexpiration and d) end-inspiration. At end-expiration, densities moved from dorsal to ventral regions. At end-inspiration, ventilation improved in prone compared to supine position.

position, alveolar inflation is greater in the nondependent lung regions. Lung weight, cardiac mass, cephalic displacement of the abdomen and the regional mechanical properties, and shape of the lung and thoracic cage are the main factors likely to influence transpulmonary pressure and the gravitational distribution of density.

Distribution of alveolar ventilation in the supine position. The distribution of alveolar ventilation follows inflation somewhat. Using CT scanning at end-inspiration and end-expiration, it is possible to measure the distribution of ventilation and alveolar recruitment (fig. 2). In sedated and paralysed ARDS patients in the supine position, the ventilation at zero PEEP is distributed preferentially to the upper lung; the ratio between the amount of ventilation in the upper and lower lung being $2.5: 1$. With increasing PEEP, the distribution of ventilation becomes progressively more homogeneous, the ratio being $\sim 1: 1$ at $20 \mathrm{cmH}_{2} \mathrm{O}$ of PEEP. This implies that modifications to regional compliance occur with PEEP, with a decrease in compliance of the upper lung due to the relatively low potential for recruitment, and an increase in the lower lung due to a relatively large amount of possible recruitment. During mechanical ventilation, the lung continuously collapses and inflates in its dependent part, especially at low levels of PEEP. At higher PEEP levels, the amount of collapsed tissue at end-expiration is decreased [13].

To conclude, in ARDS patients in supine position, alveolar ventilation is shifted preferentially to the nondependent part of the lung. Dependent lung regions continuously collapse and inflate during mechanical ventilation, but the application of PEEP causes a more homogeneous distribution of ventilation, with a reduction in regional compliance and overstretching of the nondependent lung.

Distribution of perfusion in the supine position. In normal subjects, perfusion progressively increases from nondependent to dependent lung regions [14]. However, the determinants of this perfusion gradient are not clear. Different theories have been formulated. A "gravitational" theory was proposed initially considering the relationships between pulmonary blood flow, pulmonary artery pressure $(P$ pa $), P$ A and venous return, modelled as a Starling resistor. The Starling resistor can be described as a collapsible tube (pulmonary vessels) across a closed chamber (alveoli) in which pressure may be varied. When the inflow pressure $(P \mathrm{pa})$ is lower than the chamber pressure $(P A)$, blood flow stops. When the inflow pressure is higher than the chamber pressure, flow is governed either by the difference between $P$ pa and $P$ A or by the difference between $P$ pa and venous pressure. According to this "gravitational" theory, perfusion should increase steadily down 
the lung. Other theories do not consider gravity as the main factor explaining the gravitational gradient of perfusion.

In patients with ARDS, several factors potentially influence the gravitational distribution of perfusion, including hypoxic vasoconstriction, vascular obliteration and extrinsic vessel compression. Using selective angiography, the authors have documented prevalent perfusion to the nondependent lung regions, suggesting an important role for extrinsic compression and hypoxic vasoconstriction during ARDS [15]. Indeed, recent data, obtained with more sophisticated techniques suggest prevalent perfusion to the dependent atelectatic lung regions [16].

Distribution of alveolar inflation in the prone position. As in the supine position, alveolar inflation probably depends on transpulmonary pressure. Prone positioning causes a more homogeneous distribution of transpulmonary pressure compared to the supine position [17]. The authors have observed a movement of lung densities from dorsal to ventral regions when patients were turned from supine to prone, and a more homogeneous distribution of alveolar inflation in the prone position (fig. 1). Several factors could contribute to this differential ability of the prone position to alter dorsal lung transpulmonary pressures, including a reversal of lung weight gradients, direct transmission of the weight of the heart to subjacent regions, direct transmission of the weight of abdominal contents to caudal regions of the dorsal lung and/or regional mechanical properties and shape of the chest wall and lung. All these factors are likely to produce a more homogeneous distribution of the transpulmonary pressure and consequently more homogeneous alveolar inflation.

Lung weight. The authors have found that modification of hydrostatic pressures can explain, at least in part, the redistribution of intrapulmonary gas [18]. In the supine position, hydrostatic pressures cause collapse in the dorsal (most dependent) lung regions, whilst in the prone position, the most dependent lung regions are the ventral.

Cardiac mass. More recently, the role of the cardiac mass in determining changes in densities in the prone position has been emphasised [19]. In the supine position, a considerable fraction of both lungs is located underneath the heart and, as such, is subject to compressive forces. By contrast, in the prone position, only a very small fraction of either lung would be affected.

Cephalic displacement of the abdomen. Changes in regional diaphragmatic motion occur in the prone position. In humans, the motion of the diaphragm during mechanical ventilation in the supine position is uniform, whereas when prone, most motion occurs in the nondependent (dorsal) regions [20]. However, the position of the resting diaphragm does not differ. The position and motion of the diaphragm during abdominal distension are not known. Displacement of the dome of the diaphragm into the pleural cavity has been hypothesised. Decreased intra-abdominal pressure in the prone position, thereby unloading the weight of the abdominal content, may reduce the cephalic displacement of the diaphragm and alter its position and motion compared with the supine position [21].

Regional mechanical properties and shape of the lung and chest wall. Prone positioning probably modifies the regional mechanical properties and shape of the chest wall and lung. The authors found that the distribution of alveolar inflation was more homogeneous in patients with a more "triangular" shape when supine [7]. Thus, it is evident that the shape of the lung and thorax may influence the distribution of alveolar inflation when prone. To conclude, in patients with ARDS, in the prone position, alveolar inflation is more homogeneous.

Distribution of ventilation in the prone position. Unfortunately, no data regarding the distribution of ventilation in the prone position are currently available. However, from regional inflation data, the authors infer that ventilation should redistribute from ventral (collapsed in the prone position) to dorsal regions (recruited in the prone position). Moreover, as regional inflation is more uniform in the prone position, ventilation is expected to be more uniform. To conclude, in patients with ARDS in the prone position, ventilation is probably more homogeneous and dorsally distributed.

Distribution of perfusion in prone position. To the best of the authors' knowledge, no data regarding the distribution of perfusion in the prone position are available. However, experimental evidence in dogs suggests that perfusion to dorsal regions is greater in the prone position, and that perfusion is overall more homogeneous, suggesting that mechanisms other than gravity may operate in this situation [16]. To conclude, in patients with ARDS in the prone position, perfusion is probably more homogeneous and not dependent on gravity.

\section{Effects on respiratory mechanics}

Respiratory mechanics have rarely been assessed in patients with ARDS in the prone position. Recently, the authors investigated modifications in respiratory mechanics in a group of patients with "primary" ARDS (following a direct pulmonary insult) [22]. They found that prone positioning decreased thoraco-abdominal compliance but did not affect total respiratory system compliance. The reduction in thoraco-abdominal compliance could be explained by a decrease in thoracic wall and/or diaphragmatic wall compliance. Assuming that overall compliance of the diaphragmatic wall remains unchanged in the prone position, since the intra-abdominal pressure did not change, it could be supposed that the decrease in thoracoabdominal compliance arises through a greater stiffness of the posterior, compared to the anterior, wall of the thorax when free to move. Other authors have 
shown an improvement in respiratory system compliance in the prone position, but their data mainly refer to patients with "secondary" ARDS (nonpulmonary insult) [23-25]. Interestingly, respiratory system compliance is improved when patients are returned to the supine position [22]. This indicates that structural beneficial effects can occur on the lung parenchyma in the prone position. To conclude, total respiratory system mechanics are not modified in the prone position but seem to improve after repositioning to supine.

\section{Effects on lung volume and alveolar recruitment}

The effects of prone positioning on lung volume and alveolar recruitment are unclear. Using CT scanning, the authors observed that the total amount of density was similar in supine and prone positions, suggesting no alveolar recruitment [18]. On average, lung volume and alveolar recruitment are unaffected by the posture change in patients with primary ARDS [22]. Other authors have reported alveolar recruitment, correlated to the improvement in oxygenation, in a group of patients with prevalent secondary ARDS [25]. To conclude, in patients with primary ARDS, prone positioning does not markedly influence lung volume and total alveolar recruitment. In patients with secondary ARDS, prone positioning is more likely to induce increases in lung volume and alveolar recruitment.

\section{Mechanisms of improvement in oxygenation in the prone position}

From a pathophysiological point of view, hypoxaemia in ARDS follows a reduction in the ventilation/ perfusion ratio $\left(V^{\prime} / Q^{\prime}\right)$ and the presence of a true shunt (alveolar units are not ventilated but remain perfused, $\left.V^{\prime} / Q^{\prime}=0\right)$. The combination of these two factors is called "physiological shunt". Prone positioning can improve oxygenation owing to several mechanisms that improve $V^{\prime} / Q^{\prime}$, in general, and consequently cause a reduction in physiological shunt. These include increased lung volume, redistribution of perfusion, recruitment of dorsal lung regions and a more homogeneous distribution of ventilation.

Increase in lung volume. An increase in lung volume was amongst the first mechanisms hypothesised to explain the improvements in oxygenation in the prone position [5]. Increased lung volume should be attributable to an unloading of diaphragmatic movement in the prone position, owing to a reduction in the forces opposing the passive movements of the dorsal regions. This hypothesis has not been confirmed in human studies, including those in patients with primary ARDS, since the improvement in oxygenation was not correlated with lung volume or alveolar recruitment. On the contrary, in secondary ARDS the improvement in oxygenation correlated with alveolar recruitment [25]. To conclude, the increase in lung volume and alveolar recruitment that occurs in the prone position, if present, does not entirely explain the improvement in oxygenation in primary ARDS. An increase in lung volume and alveolar recruitment may explain the improvement in oxygenation seen in secondary ARDS.

Redistribution of perfusion. This hypothesis is based on the fact that perfusion in the supine position is gravitydependent, greatest to the most dependent part of the lung, and that lung densities are also greatest in dependent regions. Thus, in the supine position, perfusion is greatest in the most diseased lung regions with a consequent increase in shunt (reduced $\left.V^{\prime} / Q^{\prime}\right)$. If the patient is turned and densities remain in the dorsal part, whilst perfusion following a gravitational gradient is increased ventrally, an improvement of $V^{\prime} / Q^{\prime}$ correlating with increased oxygenation should be expected. Unfortunately, this simple and attractive mechanism does not apply to the majority of patients with ARDS. In fact, when patients are in the prone position, although maximum perfusion is likely to remain dorsally, lung densities redistribute from dorsal to ventral regions.

Recruitment of dorsal lung with more homogeneous distribution of ventilation and perfusion. This seems to be one of the most probable causes of increased oxygenation in the prone position. In the prone position, densities in the dorsal part of the lung decrease causing more homogeneous distribution of alveolar inflation and ventilation, whilst perfusion probably remains greatest in the dorsal lung regions. Thus, $V^{\prime} / Q^{\prime}$ improves with a consequent increase in oxygenation. Recently, the authors found, in a group of patients with primary ARDS, that basal chest wall compliance and its changes played a role in determining oxygenation response to prone positioning (the lower the chest wall compliance in the supine position, the lower the improvement in oxygenation) [22]. In addition, the magnitude of the decrease in thoracoabdominal compliance observed in the prone position was related to the improvement in oxygenation. These findings, in patients with ARDS, are in line with experimental data and highlight the importance of the interactions between the rib cage, lungs and abdomen during prone positioning [21]. Moreover, the more triangular the thoracic shape in the supine position (apex on the top and base on the bottom), the greater the response in oxygenation in the prone position [7]. The improvement in oxygenation probably results from a redistribution of blood flow away from unventilated areas to regions with normal $V^{\prime} / Q^{\prime}$, most probably resulting from alveolar recruitment in previously atelectatic, but healthy and well-perfused alveoli [16]. Interestingly, in some studies, the improvement in oxygenation was partially maintained even when the patients were repositioned supine [26-28]. To conclude, redistribution of ventilation (more homogeneous and increased in the dorsal regions), associated with a more uniform distribution of perfusion, seems to be the main cause of the improvement in oxygenation seen in the prone position. The improvement can be maintained even when patients are repositioned supine. 


\section{Ventilator-associated lung injury and the prone position}

The mechanisms by which mechanical ventilation may induce or augment lung injury involve lung overdistension and repetitive opening and closing of atelectatic regions. An adverse effect on endogenous surfactant with an increased tendency for collapse of air spaces may also be involved. Why these changes occur is unclear, but high inspired oxygen fractions, high inflation pressures ("barotrauma") and large tidal volumes ("volutrauma"), and intratidal collapse and reinflation ("atelectrauma") are probably all involved. However, what is important is not the pressure per se but the delivered volume. Excessive volumes lead to high transpulmonary pressures with consequent stretch of the pulmonary tissues [29]. Recently, greater attention has been focused on intratidal collapse and reinflation, which can generate shear forces that increase capillary permeability and induce activation of inflammatory factors, leading to local and systemic inflammatory response ("biotrauma") [30].

Recent experimental studies have shown the role of prone position in attenuating and redistributing VALI $[31,32]$. In fact, lung damage, as indicated by histological abnormalities, was less in the prone than in the supine position in preinjured dogs ventilated with large tidal volumes. Moreover, while the injury in the supine position was distributed mainly to the dependent lung regions, where collapsing and reinflating phenomena are more likely to occur, in the prone position lung injury was not only reduced in total, but was more homogeneously distributed. Similar findings were obtained by other authors [33] who observed a lower incidence of pneumothorax in dogs kept prone. In conclusion, prone positioning can exert a protective effect on the mechanically ventilated injured lung.

\section{Prone position and ventilatory setting}

Positive end-expiratory pressure and recruitment manoeuvres

PEEP is commonly used to improve oxygenation in mechanically ventilated patients. PEEP may, however, cause deterioration in gas exchange when applied to patients with consolidated pneumonia [34]. Overriding hypoxic pulmonary vasoconstriction with PEEP or redistributing pulmonary blood flow away from ventilated lung units are two possible mechanisms for this adverse response. By contrast, recruitment manoeuvres using transpulmonary pressures, high enough to completely reopen atelectatic alveoli, may be beneficial before the application of PEEP when used intermittently during mechanical ventilation [2, 35]. As discussed earlier, the distribution of $P$ pl becomes more homogeneous in the prone position, leading to a more uniform distribution of ventilation and more efficient gas exchange. As transpulmonary pressures are higher ventrally than dorsally in the supine position, nondependent ventral lung regions may be relatively overexpanded when compared with dependent dorsal lung regions. This effect is likely to be exacerbated by PEEP and may contribute to the dorsal redistribution of pulmonary perfusion seen with PEEP applied in the supine position. As transpulmonary pressures are more homogeneous in the prone position, the uniform pressure distribution is unlikely to be altered by PEEP. This homogeneous pressure distribution would lead to uniform expansion of the lung in the prone position, with little redistribution of pulmonary perfusion within the lung when PEEP is administered.

In animal experiments, PEEP effectively redistributes pulmonary perfusion in the supine but not in the prone position [36]. Moreover, a greater and longerlasting effect with the time of recruitment manoeuvres has been found in experimental animals [37] and in patients with ARDS [38]. This means that lower PEEP levels are necessary to obtain the same level of oxygenation in the prone compared to the supine position.

To conclude, the application of PEEP and recruitment manoeuvres seems to be more effective in the prone than in the supine position in improving respiratory function. The effect of recruitment manoeuvres lasts longer in the prone position.

\section{Prone position and pharmacological treatment}

\section{Nitric oxide and almitrine}

Nitric oxide (NO) and prone positioning may improve oxygenation when used separately. In patients with ARDS, NO inhalation improves gas exchange by inducing vasodilatation in ventilated areas and diverting blood flow away from atelectatic nonventilated regions. Recently, several studies have investigated the effects of $\mathrm{NO}$ and prone positioning in patients with ARDS and have found a comparable improvement or a greater effect on oxygenation of prone positioning compared to NO [39-42]. However, most studies found an additive benefit when the two treatment modalities were combined [39-41]. Optimisation of alveolar recruitment increases delivery of NO to target cells, thereby improving the response to NO. The prone position should allow NO to reach previously shunted pulmonary vessels without causing alveolar overdistension. Consequently, some patients who do not respond to NO when supine, could benefit from NO inhalation in the prone position.

Other authors have suggested that the combination of NO with almitrine bismesylate would enhance the beneficial effects of prone position on oxygenation [43]. Almitrine augments hypoxic vasoconstriction redirecting blood flow to well-ventilated alveoli, but may exacerbate severe pulmonary hypertension or right ventricular failure. The suggested approach is to use NO first and add almitrine subsequently at increasing dosage [43]. However, these findings are extremely preliminary and will require further investigation.

\section{Partial liquid ventilation}

Partial liquid ventilation (PLV) facilitates the opening of collapsed, incompliant, lung regions due 
to improved surface forces induced by perfluorocarbons (PFC). Because PFC is incompressible, it may prevent complete alveolar collapse at low airway pressures, thereby improving oxygenation by decreasing intrapulmonary shunt. Limited experimental data suggest that lung recruitment could be improved by combining prone positioning and PLV [44]. However, it is not known how different doses of PFC and position would affect PFC distribution, lung mechanics and consequently oxygenation. More importantly, the combination of prone positioning and PLV may protect the lung against VALI. At present there are no clinical studies evaluating the effects of this treatment combination in patients with ARDS.

\section{Problems influencing different responses to prone positioning}

Predicting which patients will respond to prone positioning by improving oxygenation is difficult. There are three categories of patient: 1) those who do not respond $(\sim 20 \%) ; 2$ ) those who respond to prone position and who maintain any oxygenation improvement when they are returned to the supine position ( $\sim 50 \%)$; and 3 ) those who respond to prone positioning but do not maintain the improvement when they are repositioned $(\sim 30 \%)$ [27]. Several factors may predict a favourable oxygenation response on prone positioning including the morphology of the lung, the mechanical properties of the thoracic cage, and the time and aetiology of the lung injury.

\section{Morphology of the lung}

The morphology of the lung differs greatly between different populations of patients with ARDS [45]. Three main categories of patients can be classified using CT scanning: 1) patients with a "lobar" pattern in whom there were areas of lung attenuation with lobar or segmental distribution; 2) patients with a "patchy" pattern in whom there were lobar or segmental areas of lung attenuation in some parts of the lung, but lung attenuation without recognised anatomical limits in others; and 3) patients with a "diffuse" pattern in whom lung attenuation was distributed diffusely throughout the lungs. It is likely, but not proven, that patients with lobar or patchy distribution of densities are more likely to respond to prone positioning. In fact, a redistribution of densities is not likely to occur in patients with a diffuse pattern. The chest radiograph, which is simpler and safer than CT scanning, can be useful in predicting patients that are likely to respond to prone positioning. If the chest radiograph appearance is lobar or patchy, it is very likely that the CT will show a lobar or patchy pattern. By contrast, if the chest radiograph is diffuse, the $\mathrm{CT}$ scan may equally present a lobar, patchy or diffuse pattern. This means that in the presence of a chest radiograph with a diffuse pattern, a positive or negative response to prone position may be expected.

Another factor that has been found to be predictive of a positive response to prone positioning is the lung shape. Patients with a triangular thoracic shape in supine position (apex on the top and base on the bottom) enjoy greater improvements in oxygenation than those with a rectangular thoracic shape. To conclude, CT and chest radiography may be useful in predicting the response to prone positioning.

\section{Mechanical properties of the chest wall}

In primary ARDS, patients with better chest wall compliance seem to benefit more than those with decreased chest wall compliance [22]. This means that patients with an elastic anterior rib cage benefit more from prone positioning. Partitioning of the respiratory system mechanics into lung and chest wall components may be useful in predicting the response to prone positioning.

\section{Time}

Lung morphology changes with time in patients with ARDS. Radiological opacities become more homogeneous with the development of fibrosis and remodelling, whilst cysts and pseudocysts may appear [46]. Similarly remodelling of pulmonary vessels may occur. These observations increase the likelihood that the response to prone position will decrease with time.

\section{Aetiology}

Not all patients with the constellation of clinical findings that define ARDS are characterised by the same morphological and mechanical behaviour. Recently, attention has been focused on the possible differences between patients with primary ARDS and those whose lungs are diffusely injured by a process that originates elsewhere (secondary ARDS). The former patients are characterised by consolidation and appear to be less responsive to recruitment and the application of PEEP. Secondary ARDS is characterised by diffuse atelectasis that appears to be more responsive to recruitment and PEEP [47]. Since the response in oxygenation with prone positioning seems to depend on a redistribution of densities, i.e. the presence of recruitable lung, it is likely that patients with secondary ARDS will be more responsive to prone positioning.

\section{Prone position in paediatric and head-injured patients}

\section{Paediatric patients}

There are limited data regarding the effect of prone positioning in children. A recent study showed the prone position to be safe and to provide a beneficial response in paediatric patients with respiratory failure [48]. However, a second study reported an increase in oxygenation following prone positioning only in a subgroup of patients with obstructive lung disease 
[49]. In patients aged 1-5 yrs, with respiratory failure after liver transplantation, the authors observed an improvement in respiratory function following prone positioning, and this manoeuvre is used by the authors in clinical practice when indicated.

\section{Neurologically injured patients}

Patients with severe head injury are usually excluded from prone positioning, even in studies dealing with trauma victims. This is because intracranial pressure is thought to increase in the prone position and, thus, negatively affect cerebral physiology. However, in the authors' experience, head-injured patients with ARDS show improved oxygenation in the prone position and can be positioned safely, if the physicians carefully monitor intracranial pressure (preferably invasively) and the position of the neck (aligned with the spine), and ensure free abdominal movements (to reduce caval and arterial compression).

\section{Clinical studies}

\section{An overview of the literature}

Over the last $15 \mathrm{yrs}$, a series of reports have appeared in the literature dealing with the use of prone positioning as a tool to improve respiratory function in patients with ARDS. The authors have identified 31 studies up to the year 2000: 29 of which investigated adults $[4,5,18,22-28,39-43,50-63]$ and two which considered paediatric $[48,49]$ patients (not considered in the following analysis).

The majority of the studies (22 of 29) were prospective $[5,18,22-28,39-43,56-63]$, four were retrospective $[4,50-52]$, two were research letters $[54,55]$ and one was a case report [53]. The number of studies has increased over time, being two in the period 1976-1980 [4, 5], three in the period 1986-1990 [26, 54, $55]$, seven in the period 1991-1995 [18, 50-53, 56, 58], and 17 in the period 1996-2000 [22-25, 27, 28, 39-43, 57, 59-63]. The prevalence of prospective studies and the number of patients investigated in each study have also increased progressively. A total of 454 patients were involved, although the majority of studies investigated $\leqslant 20(33 \%$ investigated $<10$ patients [4, $5,18,50,52-55]$, and $45 \%<20$ patients [22, 24-26, 40, $41,43,56-58,60-63])$. Only $22 \%$ of the studies investigated $>20$ patients [23, 27, 28, 39, 42, 51, 59].

The duration of pronation was very variable between studies, ranging from $20 \mathrm{~min}$ [56] to $60 \mathrm{~h}$ [51]. However, the majority investigated the effects within $2 \mathrm{~h}(50 \%)[18,22-26,39,41-43,56,57,60]$, while $30 \%$ waited $>4 \mathrm{~h}$ and $11 \%>8 \mathrm{~h}[4,5,28,40$, $50-55,58,59,63]$. Only a minority $(7 \%)$ investigated the effects between $2-4 \mathrm{~h}[27,61,62]$. The majority of the studies investigated patients with a predominance ( $>80 \%$ of the studies) of primary ARDS [22, 27, 39-41, 43, 57] while a minority investigated patients with a predominance of secondary ARDS (11\%) [24, 56, 62, 63]. Approximately $40 \%$ investigated a group of patients with equally distributed primary and secondary ARDS (between 20-80\%) [4, 5, 18, 23, 26, $28,42,50,60,61]$. One-third of the studies $(33 \%)$ did not describe the population studied $[25,51-55,58$, 59]. Few studies investigated preselected categories of patients, although one study was performed in cardiothoracic patients [58] and two in trauma victims $[56,61]$. Repeated manoeuvres were performed in $11 \%$ $[28,56,61]$.

Except for two studies performed in pressurecontrolled ventilation [56, 60], all were performed in volume-controlled ventilation. The majority of the studies $(63 \%)$ did not describe the support used for pronation $[26,27,39,40,42,43,51-55,57-61]$. Thus, $20 \%$ did not release the abdomen $[23,56]$, whilst $80 \%$ attempted to ensure free abdominal movements $[4,5$, $18,22,24,25,28,41,50,62,63]$. All but three of the studies investigated the effects of prone position in the acute phase [28, 59, 61].

The percentage of responders at the first pronation was on average $73 \%$, a response independent of the sample size investigated $(77 \%$ responded in the studies with $\leqslant 10$ patients, $76 \%$ with $\geqslant 11$ but $\leqslant 20$ patients, and $69 \%$ with $>20$ patients). The percentage of responders was greater in studies in which the majority of patients were suffering from secondary ARDS (four studies) [24, 56, 62, 63] compared to those with primary ARDS (eight studies) [22, 25, 27, 39-41, 43, 57] $(78 \pm 4 \%$ versus $61 \pm 6 \%$, respectively; $p<0.01)$. The presence of free abdomen movement did not influence the percentage of responders. No study reported major complications related to the manoeuvre.

\section{Data from an Italian pilot study}

The pilot phase of a large, prospective, randomised, controlled, multicentre trial, designed to compare a standard therapeutic strategy, and a similar strategy, in conjunction with the daily use of prone positioning for the treatment of patients with acute respiratory failure, was performed in the period 1995-1997. Thirty-five Italian intensive care units (ICUs) were asked to apply a standardised protocol to at least two patients with predefined criteria. The entry criteria were: 1) the presence of bilateral chest infiltrates; 2) an arterial oxygen tension $\left(\mathrm{Pa}_{\mathrm{a}} \mathrm{O}_{2}\right)$ /inspiratory oxygen fraction $\left(F \mathrm{I}, \mathrm{O}_{2}\right)$ ratio $<200$ with a PEEP $\geqslant 5 \mathrm{cmH}_{2} \mathrm{O}$; and 3) no evidence of cardiac problems. The exclusion criteria were: 1) aged $<16$ yrs; 2) clinical or instrumental evidence of cardiac oedema or cerebral oedema; and 3) the presence of clinical conditions contraindicating prone positioning, such as unfixed bone fractures, haemodynamic instability and severe chest wall lesions. All patients had to be evaluated daily for a 10-day period for the presence of respiratory failure criteria (the same as the entry criteria). Patients who met these criteria were proned daily for $6 \mathrm{~h}$. Oxygenation response, tolerance of positioning and complications of the manoeuvre were recorded. Clinical data strictly related to the prone position, such as the incidence and time course of pressures, sores and nurses' workloads were also assessed.

Seventy-three patients (45 male and 28 female; 51 with primary ARDS) were enrolled. The mean \pm SD 
Table 1. - Manoeuvre-related complications

\begin{tabular}{lcc}
\hline Complication & Events & Percentage \\
\hline $\begin{array}{l}\text { Airway obstruction } \\
\quad \text { secretion) }\end{array}$ & $102 / 772$ & 13 \\
$\begin{array}{l}\text { Transient oxygen } \\
\quad \text { desaturation }\end{array}$ & $97 / 764$ & 13 \\
Arrhythmias & $16 / 773$ & 2 \\
Hypotension & $15 / 773$ & 1.9 \\
Vomiting & $12 / 773$ & 1.6 \\
Accidental loss of central & $5 / 775$ & 0.6 \\
$\quad$ venous catheter & $3 / 772$ & 0.4 \\
Accidental extubation & & 0.2 \\
Accidental loss of thoracic & $2 / 671$ & \\
$\quad$ or abdominal drains & & \\
\hline
\end{tabular}

age was $51 \pm 17$ yrs, simplified acute physiology score at entry was $38 \pm 11$ and time before enrolment was $2.8 \pm 3.2$ days. The clinical data at entry were (mean \pm SD) $P$ a $, \mathrm{O}_{2} / F \mathrm{I}, \mathrm{O}_{2}, \quad 123 \pm 42 ; \quad P \mathrm{a}, \mathrm{CO}_{2}, \quad 6.0 \pm 1.9 \mathrm{kPa} \quad(45 \pm$ $14 \mathrm{mmHg}$; $\mathrm{pH}, 7.39 \pm 0.09$; PEEP, $10 \pm 3 \mathrm{cmH}_{2} \mathrm{O}$; mean airway pressure, $18 \pm 5 \mathrm{cmH}_{2} \mathrm{O}$; peak airway pressure, $33 \pm 7 \mathrm{cmH}_{2} \mathrm{O}$; minute volume, $10.9 \pm 2.9 \mathrm{~L}$; and tidal volume, $680 \pm 115 \mathrm{~mL}$. The total number of pronations was 390 . During the first day after the admission to the ICU, 71 patients were placed in the prone position for $6.2 \pm 1.2 \mathrm{~h}$. After the first hour of prone positioning, the $\mathrm{Pa}, \mathrm{O}_{2} / F \mathrm{I}, \mathrm{O}_{2}$ of $76 \%$ of the patients had increased by $>2.7 \mathrm{kPa}(20 \mathrm{mmHg}$; responders) with an increase of $10.4 \pm 7.0 \mathrm{kPa}(78 \pm$ $53 \mathrm{mmHg}$ ). The proportion of responders increased to $85 \%$ after $6 \mathrm{~h}$ of prone positioning. The authors' assessment of the effect of this manoeuvre $12 \mathrm{~h}$ later, in the supine position, revealed that oxygenation was improved $\left(\mathrm{Pa}, \mathrm{O}_{2} / F \mathrm{I}, \mathrm{O}_{2}: 151 \pm 39\right.$ versus $\left.123 \pm 42 ; \mathrm{p}<0.01\right)$.

The improvement in oxygenation, expressed as $\Delta \mathrm{Pa}_{\mathrm{a}} \mathrm{O}_{2} / F \mathrm{I}, \mathrm{O}_{2}$ was greater in patients with secondary compared to primary ARDS during the 10-day period $(77 \pm 24$ versus $52 \pm 12 ; \mathrm{p}<0.01)$. The percentage of manoeuvres in which patient/ventilator dyssynchrony occurred was $13 \%$, while in $39 \%$ it was necessary to add sedation and in $24 \%$ it was necessary to add muscular relaxants. As shown in table 1, manoeuvrerelated complications and severe life-threatening complications were extremely rare. More importantly, $76 \%$ of the patients developed pressure sores, the majority $(63 \%)$ of which were severe. Each patient, at the end of the 10-day study period, presented on average 2.9 sores and the number of severe sores per patient was 1.8. The development of sores was principally on the pelvis $(46 \%)$, followed by the thorax $(21 \%)$, legs $(19 \%)$, arms $(15 \%)$, head $(7 \%)$ and other sites $(7 \%)$. However, severe sores were prevalent on the pelvis $(41 \%)$ and legs $(42 \%)$. The main predictor

Table 2.-Synopsis of current trials of prone positioning

\begin{tabular}{|c|c|c|c|c|c|c|c|c|c|}
\hline First author [ref.] & Type & Year & Patient $\mathrm{n}$ & $\begin{array}{c}\text { Prevalent } \\
\text { disease }\end{array}$ & Support & $\begin{array}{l}\text { Improved } \\
\text { patients } \%\end{array}$ & $\begin{array}{c}\mathrm{Pa}_{\mathrm{a}, \mathrm{O}_{2}} \\
\text { supine } \mathrm{kPa}\end{array}$ & $\begin{array}{c}\mathrm{Pa}_{\mathrm{a}, \mathrm{O}_{2}} \\
\text { prone } \mathrm{kPa}\end{array}$ & $\begin{array}{c}\text { Survival } \\
\%\end{array}$ \\
\hline PIEHL [4] & $\mathrm{R}$ & 1976 & 5 & M & FA & 100 & $9.6 \pm 1.7$ & $14.1 \pm 1.2$ & 60 \\
\hline DOUGLAS [5] & $\mathrm{P}$ & 1977 & 6 & M & FA & 100 & $8.4 \pm 1.3$ & $18.4 \pm 8.9$ & 50 \\
\hline GATTINONI [18] & $\mathrm{P}$ & 1991 & 10 & M & FA & 30 & $9.3 \pm 0.9$ & $15.4 \pm 4.3$ & 60 \\
\hline Pelosi [22] & $\mathrm{P}$ & 1998 & 17 & PR & FA & 76 & $13.3 \pm 3.5$ & $16.6 \pm 3.6$ & 59 \\
\hline BLANCH [23] & $\mathrm{P}$ & 1997 & 23 & M & NS & 67 & $9.3 \pm 3.6^{\#}$ & $12.0 \pm 7.0$ & 52 \\
\hline SERVILLO [24] & $\mathrm{P}$ & 1997 & 12 & $\mathrm{~S}$ & FA & 83 & $16.4 \pm 2.9$ & $20.3 \pm 2.3$ & 42 \\
\hline GUERIN [25] & $\mathrm{P}$ & 1999 & 12 & $\mathrm{U}$ & FA & 67 & $18.1 \pm 2.3$ & $27.1 \pm 3.2$ & 58 \\
\hline LANGER [26] & $\mathrm{P}$ & 1988 & 13 & M & $\mathrm{U}$ & 62 & $9.2 \pm 1.1$ & $14.8 \pm 2.7$ & 54 \\
\hline CHATTE [27] & $\mathrm{P}$ & 1997 & 32 & PR & $\mathrm{U}$ & 31 & $13.3 \pm 4.8$ & $23.9 \pm 4.9$ & 44 \\
\hline GATTINONI [28] & $\mathrm{P}$ & 1997 & 54 & M & FA & 76 & $16.4 \pm 5.3^{\#}$ & $25.9 \pm 9.3$ & 54 \\
\hline GERMANN [39] & $\mathrm{P}$ & 1998 & 47 & PR & $\mathrm{U}$ & 74 & $15.3^{\circ}$ & 21.9 & 91 \\
\hline PAPAZIAN [40] & $\mathrm{P}$ & 1998 & 14 & PR & $\mathrm{U}$ & 64 & $17.0 \pm 5.9^{\#}$ & $25.7 \pm 11.0$ & 85 \\
\hline MARTINEZ [41] & $\mathrm{P}$ & 1999 & 14 & PR & FA & 41 & $14.6 \pm 7.3^{\#}$ & $21.4 \pm 11.8$ & 43 \\
\hline DUPONT [42] & $\mathrm{P}$ & 2000 & 27 & $\mathrm{M}$ & U & 74 & $12.2 \pm 3.5^{\#}$ & $22.5 \pm 10.1$ & 37 \\
\hline JOLLIET [43] & $\mathrm{P}$ & 1997 & 12 & PR & $\mathrm{U}$ & 58 & $8.2 \pm 0.9$ & $9.6 \pm 1.9$ & 33 \\
\hline ALBERT [50] & $\mathrm{R}$ & 1993 & 9 & M & FA & 78 & $8.4 \pm 2.0$ & $30.7 \pm 16.1$ & \\
\hline WALZ [51] & $\mathrm{R}$ & 1992 & 16 & $\mathrm{U}$ & $\mathrm{U}$ & 100 & & & \\
\hline SHICHINOHE [52] & $\mathrm{R}$ & 1991 & 7 & $\mathrm{U}$ & $\mathrm{U}$ & 100 & & & \\
\hline THulig [53] & $\mathrm{C}$ & 1991 & 1 & $\mathrm{U}$ & $\mathrm{U}$ & 100 & 8.9 & 11.8 & \\
\hline FALLER [54] & $\mathrm{L}$ & 1988 & 3 & $\mathrm{U}$ & $\mathrm{U}$ & 100 & $13.7 \pm 3.3$ & $26.2 \pm 3.9$ & \\
\hline MANARA [55] & $\mathrm{L}$ & 1987 & 4 & $\mathrm{U}$ & $\mathrm{U}$ & 75 & $39.9 \pm 7.0^{+}$ & $19.0 \pm 2.0$ & 25 \\
\hline PAPPERT [56] & $\mathrm{P}$ & 1994 & 12 & $\mathrm{~S}$ & NS & 56 & $9.8 \pm 1.5$ & $16.4 \pm 3.9$ & 58 \\
\hline VOLLMAN [57] & $\mathrm{P}$ & 1996 & 15 & PR & $\mathrm{U}$ & 60 & $11.6 \pm 1.9$ & $13.6 \pm 4.4$ & \\
\hline BRUSSEL [58] & $\mathrm{P}$ & 1993 & 10 & $\mathrm{U}$ & $\mathrm{U}$ & 74 & $9.7 \pm 3.9$ & $13.4 \pm 5.2$ & 90 \\
\hline VOGGENREITER [59] & $\mathrm{P}$ & 1999 & 22 & $\mathrm{U}$ & $\mathrm{U}$ & 90 & $19.8 \pm 1.1^{\#}$ & $39.5 \pm 4.0$ & 82 \\
\hline WENZ [60] & $\mathrm{P}$ & 2000 & 10 & M & $\mathrm{U}$ & 80 & $20.0 \pm 4.9$ & $29.3 \pm 2.3$ & 90 \\
\hline FRIEDRICH [61] & $\mathrm{P}$ & 1996 & 20 & M & $\mathrm{U}$ & 60 & $12.9 \pm 0.5$ & $20.2 \pm 2.0$ & 90 \\
\hline MURE [62] & $\mathrm{P}$ & 1997 & 13 & $\mathrm{~S}$ & FA & 92 & $69.7 \pm 18.1^{+}$ & $34.3 \pm 18.8$ & 69 \\
\hline FLAATTEN[63] & $\mathrm{P}$ & 1998 & 14 & S & FA & 90 & $11.7 \pm 0.8$ & $18 \pm 1.4$ & 57 \\
\hline
\end{tabular}

P: prospective; R: retrospective; C: case report; L: letter; M: primary/secondary acute respiratory distress syndrome (ARDS) between 20-80\%; PR: $>80 \%$ primary ARDS; S: $>80 \%$ secondary ARDS; U: data not available; FA: free abdomen; NS: not supported; $\mathrm{Pa}, \mathrm{O}_{2}$ : arterial oxygen pressure. ${ }^{\#}$ : arterial oxygen tension/inspiratory oxygen fraction; ${ }^{\uparrow}:$ median; ${ }^{+}$: alveolararterial oxygen pressure difference. 
for pressure sore formation was the number of pronations $(6.2 \pm 2.8$ pronations in patients with sores versus $3.6 \pm 2.6$ pronations in patients without sores; $\mathrm{p}<0.05)$. Different supports were used in the supine and prone positions. In the majority of patients air mattresses $(39 \%)$ and water mattress covers $(24 \%)$ were used. The devices used during pronation were pillows $(30 \%)$, sheet rolls $(21 \%)$, and water pillows $(11 \%)$. The overall mortality at discharge from the ICU was $51 \%$, which was not significantly different between primary and secondary ARDS. The stay in the ICU was similar in survivors and nonsurvivors (17.8 \pm 11.6 versus $17.8 \pm 11.4$ days).

\section{Conclusion}

From these data (summarised in table 2) and the physiological findings presented and discussed earlier, the authors conclude: 1) prone positioning improves oxygenation in the majority of patients $(\sim 70-80 \%)$ with ARDS; 2) prone positioning does not negatively affect and may improve respiratory mechanics; 3 ) the improvement in oxygenation and respiratory mechanics is partially maintained after returning the patient to the supine position; 4) the effects on oxygenation are reduced after the first week of mechanical ventilation; 5) the effects of prone positioning on respiratory function may be related to the specific aetiology of ARDS; 6) it is possible that prone positioning is more effective in secondary than in primary ARDS, but larger studies are needed to confirm this hypothesis; 7) manoeuvre-related complications are rare, but pressure sores are frequent and mainly related to the number of pronations; 8 ) the supports used to prone, and during positioning, are not standardised among centres; and 9) the mortality of ARDS remains high even if patients are continuously proned.

For the future, formal trials in a representative sample of patients are needed to establish whether prone positioning can improve survival, together with more physiopathological knowledge to identify which categories of patients can benefit effectively from prone position, the optimal ventilatory setting to be selected before, during and after positioning, the duration and frequency of positioning, and a standardisation of the manoeuvre.

\section{References}

1. The Acute Respiratory Distress Syndrome Network. Ventilation with lower tidal volumes as compared with traditional tidal volumes for acute respiratory distress syndrome. N Engl J Med 2000; 342: 1301-1308.

2. Amato MBP, Barbas CSV, Medeiros DM, et al. Effect of a protective-ventilation strategy on mortality in the acute respiratory distress syndrome. $N$ Engl J Med 1998; 338: 347-354.

3. Bryan AC. Comments of a devil's advocate. Am Rev Respir Dis 1974; 110: 143-144.

4. Piehl MA, Brown RS. Use of extreme position changes in acute respiratory failure. Crit Care Med 1976; 4: 13-14.

5. Douglas WW, Rehder K, Beynen FM, Sessler AD, Marsh HM. Improved oxygenation in patients with acute respiratory failure: the prone position. Am Rev Respir Dis 1977; 115: 559-566.

6. $\quad$ Albert RK. One good turn. Intensive Care Med 1994; 20: $247-248$

7. Gattinoni L, Pelosi P, Valenza F, Mascheroni D. Patient positioning in acute respiratory failure. In: Tobin M, ed. Principles and Practice of Mechanical Ventilation. New York, McGraw-Hill, 1994; pp. 1067-1076.

8. Pelosi P, D'Andrea L, Vitale G, Pesenti A, Gattinoni L. Vertical gradient of regional lung inflation in adult respiratory distress syndrome. Am J Respir Crit Care Med 1994; 149: 8-13.

9. Hyatt RE, Bar-Yishay E, Abel MD. Influence of the heart on the vertical gradient of transpulmonary pressure in dogs. J Appl Physiol 1985; 58: 52-57.

10. Malbouisson LM, Busch CJ, Puybasset L, et al. Role of the heart in the loss of aeration characterizing lower lobes in acute respiratory distress syndrome. Am J Respir Crit Care Med 2000; 161: 2005-2012.

11. Froese AB, Bryan AC. Effects of anesthesia and paralysis on diaphragmatic mechanics in man. Anesthesiology 1974; 41: 242-255.

12. Margulies SS, Rodarte JR. Shape of the chest wall in the prone and supine anesthetized dog. $J$ Appl Physiol 1990; 68: 1970-1978.

13. Gattinoni L, Pelosi P, Crotti S, Valenza F. Effects of positive end-expiratory pressure on regional distribution of tidal volume and recruitment in adult respiratory distress syndrome. Am J Respir Crit Care Med 1995; 151: 1807-1814.

14. West JB, Dolley CT, Naimark A. Distribution of blood flow in isolated lungs: relation to vascular and alveolar pressures. J Appl Physiol 1964; 19: 713-724.

15. Vesconi S, Rossi GP, Pesenti A, Fumagalli R, Gattinoni L. Pulmonary microthrombosis in severe adult respiratory distress syndrome. Crit Care Med 1988; 16: 111-113.

16. Lamm WJE, Graham MM, Albert RK. Mechanism by which the prone position improves oxygenation in acute lung injury. Am J Respir Crit Care Med 1994; 150: 184-193.

17. Mutoh T, Guest RJ, Lamm WJE, Albert RK. Prone position alters the effect of volume overload on regional pleural pressures and improves hypoxemia in pigs in vivo. Am Rev Respir Dis 1992; 146: 300-306.

18. Gattinoni L, Pelosi P, Vitale G, Pesenti A, D'Andrea L, Mascheroni D. Body position changes redistribute lung computed-tomographic density in patients with acute respiratory failure. Anesthesiology 1991; 74: 1523.

19. Albert RK, Hubmayr RD. The prone position eliminates compression of the lung by the heart. Am J Respir Crit Care Med 2000; 161: 1660-1665.

20. Krayer S, Rehder K, Vettermann J, Didier P, Ritman EL. Position and motion of the human diaphragm during anesthesia-paralysis. Anesthesiology 1989; 70: 891-898.

21. Mure M, Glenny RW, Domino KB, Hlastala MP. Pulmonary gas exchange improves in the prone position with abdominal distension. Am J Respir Crit Care Med 1998; 157: 1785-1790.

22. Pelosi P, Tubiolo D, Mascheroni D, et al. Effects of 
the prone position on respiratory mechanics and gas exchange during acute lung injury. Am J Respir Crit Care Med 1998; 157: 387-393.

23. Blanch L, Mancebo J, Perez M, et al. Short-term effects of prone position in critically ill patients with acute respiratory distress syndrome. Intensive Care Med 1997; 23: 1033-1039.

24. Servillo G, Roupie E, De Robertis E, et al. Effects of ventilation in ventral decubitus position on respiratory mechanics in adult respiratory distress syndrome. Intensive Care Med 1997; 23: 1219-1224.

25. Guerin C, Badet M, Rosselli S, et al. Effects of prone position on alveolar recruitment and oxygenation in acute lung injury. Intensive Care Med 1999; 25: 12221230.

26. Langer M, Mascheroni D, Marcolin R, Gattinoni L. The prone position in ARDS patients: a clinical study. Chest 1988; 94: 103-107.

27. Chatte G, Sab J, Dubois J, Sirodot M, Gaussorgues P, Robert D. Prone position in mechanically ventilated patients with severe acute respiratory failure. $A m J$ Respir Crit Care Med 1997; 155: 473-478.

28. Gattinoni L, Tognoni L, Brazzi L, et al. Ventilation in the prone position. The prone-supine study collaborative group. Lancet 1997; 350: 815.

29. Dreyfuss D, Basset G, Soler P, Saumon G. Intermittent positive-pressure hyperventilation with high inflation pressures produces pulmonary microvascular injury in rats. Am Rev Respir Dis 1985; 132: 880-884.

30. Ranieri VM, Suter PM, Tortorella C, et al. Effect of mechanical ventilation on inflammatory mediators in patients with acute respiratory distress syndrome. JAMA 1999; 282: 54-61.

31. Broccard AF, Shapiro RS, Schmitz LL, Ravenscraft SA, Marini JJ. Influence of prone position on the extent and distribution of lung injury in a high tidal volume oleic acid model of acute respiratory distress syndrome. Crit Care Med 1997; 25: 16-27.

32. Broccard AF, Shapiro RS, Schmitz LL, Adams AB, Nahum A, Marini JJ. Prone positioning attenuates and redistributes ventilator-induced lung injury in dogs. Crit Care Med 2000; 28: 295-303.

33. Du HL, Yamada Y, Orii R, et al. Beneficial effects of the prone position on the incidence of barotrauma in oleic acid-induced lung injury under continuous positive pressure ventilation. Acta Anesth Scand 1997; 41: 701-707.

34. Mink SN, Light RB, Cooligan T. Effect of PEEP on gas exchange and pulmonary perfusion in canine lobar pneumonia. J Appl Physiol 1981; 50: 517-523.

35. Lachmann B. Open up the lung and keep the lung open. Intensive Care Med 1992; 18: 319-321.

36. Walther SM, Domino KB, Glenny RW, Hlastala MP. Positive end-expiratory pressure redistributes perfusion to dependent lung regions in supine but not in prone lambs. Crit Care Med 1999; 27: 37-45.

37. Cakar N, van der Kloot T, Youngblood M, Adams A, Nahum A. Oxygenation response to a recruitment maneuver during supine and prone positions in an oleic acid-induced lung injury model. Am J Respir Crit Care Med 2000; 161: 1949-1956.

38. Pelosi P, Bottino N, Caironi P, et al. Sigh in acute respiratory distress syndrome: effects in supine and in prone position. Intensive Care Med 2000; 26: S263.

39. Germann P, Poschl G, Leitner C, et al. Additive effect of nitric oxide inhalation on the oxygenation benefit of the prone position in the adult respiratory distress syndrome. Anesthesiology 1998; 89: 1401-1406.

40. Papazian L, Bregeon F, Gaillat F, et al. Respective and combined effects of prone position and inhaled nitric oxide in patients with acute respiratory distress syndrome. Am J Respir Crit Care Med 1998; 157: 580585.

41. Martinez M, Diaz E, Joseph D, et al. Improvement in oxygenation by prone position and nitric oxide in patients with acute respiratory distress syndrome. Intensive Care Med 1999; 25: 29-36.

42. Dupont H, Mentec H, Cheval C, Moine P, Fierobe L, Timsit J-F. Short-term effect of inhaled nitric oxide and prone positioning on gas exchange in patients with severe acute respiratory distress syndrome. Crit Care Med 2000; 28: 304-308.

43. Jolliet P, Bulpa P, Ritz M, Ricou B, Lopez J, Chevrolet JC. Additive beneficial effects of the prone position, nitric oxide, and almitrine bismesylate on gas exchange and oxygen transport in acute respiratory distress syndrome. Crit Care Med 1997; 25: 786-794.

44. Max M, Kuhlen R, Lopez F, Reyle-Hahn SM, Baumert JH, Roissaint R. Combining partial liquid ventilation and prone position in experimental acute lung injury. Anesthesiology 1999; 91: 796-803.

45. Puybasset L, Cluzel P, Gusman P, et al. Regional distribution of gas and tissue in acute respiratory distress syndrome. I. Consequences for lung morphology. Intensive Care Med 2000; 26: 857-869.

46. Gattinoni L, Bombino $\mathrm{M}$, Pelosi $\mathrm{P}$, et al. Lung structure and function in different stages of severe adult respiratory distress syndrome. JAMA 1994; 271 : 1772-1779.

47. Goodman LR, Fumagalli R, Tagliabue $\mathrm{P}$, et al. Adult respiratory distress syndrome due to pulmonary and extrapulmonary causes: CT, clinical and functional correlations. Radiology 1999; 213: 545-552.

48. Curley MAQ, Thompson JE, Arnold JH. The effects of early and repeated prone positioning in pediatric patients with acute lung injury. Chest 2000; 118: 156163.

49. Numa AH, Hammer J, Newth CJL. Effect of prone and supine positions on functional residual capacity, oxygenation, and respiratory mechanics in ventilated infants and children. Am J Respir Crit Care Med 1997; 156: $1185-1189$.

50. Albert RK. New ideas in treatment of ARDS. In: Yearbook of Intensive Care and Emergency Medicine. Berlin, Springer-Verlag, 1993; pp. 135-147.

51. Walz M, Muhr G. Continuously alternating prone and supine positioning in acute lung failure. Chirurg 1992; 63: 931-937.

52. Shichinohe Y, Ujike Y, Kurihara M, et al. Respiratory care with prone position for diffuse atelectasis in critically ill patients. Kokyn-to-Junkan 1991; 39: 51-55.

53. Thulig B, Hachenberg T, Wendt M, Wiesman W, Sulkowski V. Artificial respiration in the prone position in a case of acute respiratory distress syndrome. Anesthesiol Intensivmed Notfallmed Schmerzther 1991; 26: 196-198.

54. Faller JP, Feissel M, Kara A, Camelot R, Simon G. La ventilation en procubitus dans les syndromes de detresse respiratorie aigue $\mathrm{d}^{\prime}$ evolution severe. [Ventilation in prone position in acute respiratory distress syndrome of severe course. 3 cases]. Presse Med 1988; 22: 1154

55. Manara AR, Fromant SM, Canter C, Park GR. 
Improving oxygenation using the prone position. Intensive Care Med 1987; 13: 218-219.

56. Pappert D, Roissant R, Slama K, Gruning T, Falke $\mathrm{KJ}$. Influence of positioning on ventilation-perfusion relationship in severe adult respiratory distress syndrome. Chest 1994; 106: 1511-1516.

57. Vollman KM, Bander JJ. Improved oxygenation utilizing a prone positioner in patients with acute respiratory distress syndrome. Intensive Care Med 1996; 22: 1105-1111.

58. Brussel $\mathrm{T}$, Hachenberg $\mathrm{T}$, Roos $\mathrm{N}$, Lemzem $\mathrm{H}$, Konertz W, Lawin P. Mechanical ventilation in the prone position for acute respiratory failure after cardiac surgery. J Cardiothorac Vasc Anesth 1993; 7: 541-546.

59. Voggenreiter G, Neudeck F, Aufmkolk M, et al. Intermittent prone positioning in the treatment of severe and moderate posttraumatic lung injury. Crit Care Med 1999; 27: 2375-2382.

60. Wenz M, Hoffmann B, Bohlender J, Kaczmarczyk G. Angiotensin II formation and endothelin clearance in ARDS patients in supine and prone position. Intensive Care Med 2000; 26: 292-298.

61. Friedrich P, Krafft P, Hochleuthner H, Mauritz W. The effects of long-term prone positioning in patients with trauma induced adult respiratory distress syndrome. Anesth Analg 1996; 83: 1206-1211.

62. Mure M, Martling C, Lindahl SGE. Dramatic effect on oxygenation in patients with severe acute lung insufficiency treated in the prone position. Crit Care Med 1997; 25: 1539-1544.

63. Flaatten H, Aardal S, Hevroy O. Improved oxygenation using the prone position in patients with ARDS. Acta Anaesthesiol Scand 1998; 42: 329-334. 